\title{
An Existence Result for Nonlinear Fractional Differential Equations on Banach Spaces
}

\author{
Mouffak Benchohra, ${ }^{1}$ Alberto Cabada, ${ }^{2}$ and Djamila Seba ${ }^{3}$ \\ ${ }^{1}$ Laboratoire de Mathématiques, Université de Sidi Bel-Abbès, BP 89, 22000 Sidi Bel-Abbès, Algeria \\ 2 Departamento de Analisis Matematico, Facultad de Matematicas, Universidad de Santiago de Compostela, \\ 15782, Santiago de Compostela, Spain \\ ${ }^{3}$ Département de Mathématiques, Université de Boumerdès, Avenue de l'Indépendance, \\ 35000 Boumerdès, Algeria
}

Correspondence should be addressed to Mouffak Benchohra, benchohra@univ-sba.dz

Received 30 January 2009; Revised 23 March 2009; Accepted 15 May 2009

Recommended by Juan J. Nieto

The aim of this paper is to investigate a class of boundary value problem for fractional differential equations involving nonlinear integral conditions. The main tool used in our considerations is the technique associated with measures of noncompactness.

Copyright (c) 2009 Mouffak Benchohra et al. This is an open access article distributed under the Creative Commons Attribution License, which permits unrestricted use, distribution, and reproduction in any medium, provided the original work is properly cited.

\section{Introduction}

The theory of fractional differential equations has been emerging as an important area of investigation in recent years. Let us mention that this theory has many applications in describing numerous events and problems of the real world. For example, fractional differential equations are often applicable in engineering, physics, chemistry, and biology. See Hilfer [1], Glockle and Nonnenmacher [2], Metzler et al. [3], Podlubny [4], Gaul et al. [5], among others. Fractional differential equations are also often an object of mathematical investigations; see the papers of Agarwal et al. [6], Ahmad and Nieto [7], Ahmad and OteroEspinar [8], Belarbi et al. [9], Belmekki et al [10], Benchohra et al. [11-13], Chang and Nieto [14], Daftardar-Gejji and Bhalekar [15], Figueiredo Camargo et al. [16], and the monographs of Kilbas et al. [17] and Podlubny [4].

Applied problems require definitions of fractional derivatives allowing the utilization of physically interpretable initial conditions, which contain $y(0), y^{\prime}(0)$, and so forth. the same requirements of boundary conditions. Caputo's fractional derivative satisfies these demands. For more details on the geometric and physical interpretation for fractional derivatives of both the Riemann-Liouville and Caputo types, see [18, 19]. 
In this paper we investigate the existence of solutions for boundary value problems with fractional order differential equations and nonlinear integral conditions of the form

$$
\begin{gathered}
{ }^{c} D^{r} y(t)=f(t, y(t)), \quad \text { for each } t \in J=[0, T], \\
y(0)-y^{\prime}(0)=\int_{0}^{T} g(s, y(s)) d s, \\
y(T)+y^{\prime}(T)=\int_{0}^{T} h(s, y(s)) d s,
\end{gathered}
$$

where ${ }^{c} D^{r}, 1<r \leq 2$ is the Caputo fractional derivative, $f, g$, and $h: J \times E \rightarrow E$ are given functions satisfying some assumptions that will be specified later, and $E$ is a Banach space with norm $\|\cdot\|$.

Boundary value problems with integral boundary conditions constitute a very interesting and important class of problems. They include two, three, multipoint, and nonlocal boundary value problems as special cases. Integral boundary conditions are often encountered in various applications; it is worthwhile mentioning the applications of those conditions in the study of population dynamics [20] and cellular systems [21].

Moreover, boundary value problems with integral boundary conditions have been studied by a number of authors such as, for instance, Arara and Benchohra [22], Benchohra et al. [23, 24], Infante [25], Peciulyte et al. [26], and the references therein.

In our investigation we apply the method associated with the technique of measures of noncompactness and the fixed point theorem of Mönch type. This technique was mainly initiated in the monograph of Bana and Goebel [27] and subsequently developed and used in many papers; see, for example, Bana and Sadarangoni [28], Guo et al. [29], Lakshmikantham and Leela [30], Mönch [31], and Szufla [32].

\section{Preliminaries}

In this section, we present some definitions and auxiliary results which will be needed in the sequel.

Denote by $C(J, E)$ the Banach space of continuous functions $J \rightarrow E$, with the usual supremum norm

$$
\|y\|_{\infty}=\sup \{\|y(t)\|, t \in J\} .
$$

Let $L^{1}(J, E)$ be the Banach space of measurable functions $y: J \rightarrow E$ which are Bochner integrable, equipped with the norm

$$
\|y\|_{L^{1}}=\int_{0}^{T}\|y(s)\| d s
$$


Let $L^{\infty}(J, E)$ be the Banach space of measurable functions $y: J \rightarrow E$ which are bounded, equipped with the norm

$$
\|y\|_{L^{\infty}}=\inf \{c>0:\|y(t)\| \leq c \text {, a.e. } t \in J\} .
$$

Let $A C^{1}(J, E)$ be the space of functions $y: J \rightarrow E$, whose first derivative is absolutely continuous.

Moreover, for a given set $V$ of functions $v: J \rightarrow E$ let us denote by

$$
\begin{gathered}
V(t)=\{v(t), v \in V\}, \quad t \in J, \\
V(J)=\{v(t): v \in V\}, \quad t \in J .
\end{gathered}
$$

Now let us recall some fundamental facts of the notion of Kuratowski measure of noncompactness.

Definition 2.1 (see [27]). Let $E$ be a Banach space and $\Omega_{E}$ the bounded subsets of $E$. The Kuratowski measure of noncompactness is the map $\alpha: \Omega_{E} \rightarrow[0, \infty]$ defined by

$$
\alpha(B)=\inf \left\{\epsilon>0: B \subseteq \bigcup_{i=1}^{n} B_{i} \text { and } \operatorname{diam}\left(B_{i}\right) \leq \epsilon\right\} ; \quad \text { here } B \in \Omega_{E} .
$$

\section{Properties}

The Kuratowski measure of noncompactness satisfies some properties (for more details see [27]).
(a) $\alpha(B)=0 \Leftrightarrow \bar{B}$ is compact ( $B$ is relatively compact).
(b) $\alpha(B)=\alpha(\bar{B})$.
(c) $A \subseteq B \Rightarrow \alpha(A) \leq \alpha(B)$.
(d) $\alpha(A+B) \leq \alpha(A)+\alpha(B)$.
(e) $\alpha(c B)=|c| \alpha(B) ; c \in \mathbb{R}$.
(f) $\alpha(\operatorname{coB})=\alpha(B)$.

Here $\bar{B}$ and $c o B$ denote the closure and the convex hull of the bounded set $B$, respectively.

For completeness we recall the definition of Caputo derivative of fractional order.

Definition 2.2 (see [17]). The fractional order integral of the function $h \in L^{1}([a, b])$ of order $r \in \mathbb{R}_{+}$; is defined by

$$
I_{a}^{r} h(t)=\frac{1}{\Gamma(r)} \int_{a}^{t} \frac{h(s)}{(t-s)^{1-r}} d t
$$


where $\Gamma$ is the gamma function. When $a=0$, we write $I^{r} h(t)=\left[h * \varphi_{r}\right](t)$, where

$$
\varphi_{r}(t)=\frac{t^{r-1}}{\Gamma(r)} \text { for } t>0
$$

$\varphi_{r}(t)=0$ for $t \leq 0$, and $\varphi_{r} \rightarrow \delta(t)$ as $r \rightarrow 0$.

Here $\delta$ is the delta function.

Definition 2.3 (see [17]). For a function $h$ given on the interval $[a, b]$, the Caputo fractionalorder derivative of $h$, of order $r>0$, is defined by

$$
{ }^{c} D_{a^{+}}^{r} h(t)=\frac{1}{\Gamma(n-r)} \int_{a}^{t} \frac{h^{(n)}(s) d s}{(t-s)^{1-n+r}} .
$$

Here $n=[r]+1$ and $[r]$ denotes the integer part of $r$.

Definition 2.4. A map $f: J \times E \rightarrow E$ is said to be Carathéodory if

(i) $t \mapsto f(t, u)$ is measurable for each $u \in E$;

(ii) $u \mapsto f(t, u)$ is continuous for almost each $t \in J$.

For our purpose we will only need the following fixed point theorem and the important Lemma.

Theorem 2.5 (see $[31,33])$. Let $D$ be a bounded, closed and convex subset of a Banach space such that $0 \in D$, and let $N$ be a continuous mapping of $D$ into itself. If the implication

$$
V=\overline{c o} N(V) \quad \text { or } \quad V=N(V) \cup\{0\} \Longrightarrow \alpha(V)=0
$$

holds for every subset $V$ of $D$, then $N$ has a fixed point.

Lemma 2.6 (see [32]). Let $D$ be a bounded, closed, and convex subset of the Banach space $C(J, E)$, $G$ a continuous function on $J \times J$, and a function $f: J \times E \rightarrow E$ satisfies the Carathéodory conditions, and there exists $p \in L^{1}\left(J, \mathbb{R}_{+}\right)$such that for each $t \in J$ and each bounded set $B \subset E$ one has

$$
\lim _{k \rightarrow 0^{+}} \alpha\left(f\left(J_{t, k} \times B\right)\right) \leq p(t) \alpha(B) ; \quad \text { where } J_{t, k}=[t-k, t] \cap J
$$

If $V$ is an equicontinuous subset of $D$, then

$$
\alpha\left(\left\{\int_{J} G(s, t) f(s, y(s)) d s: y \in V\right\}\right) \leq \int_{J}\|G(t, s)\| p(s) \alpha(V(s)) d s
$$




\section{Existence of Solutions}

Let us start by defining what we mean by a solution of the problem (1.1).

Definition 3.1. A function $y \in A C^{1}(J, E)$ is said to be a solution of (1.1) if it satisfies (1.1). problem

Let $\sigma, \rho_{1}, \rho_{2}: J \rightarrow E$ be continuous functions and consider the linear boundary value

$$
\begin{gathered}
{ }^{c} D^{r} y(t)=\sigma(t), \quad t \in J, \\
y(0)-y^{\prime}(0)=\int_{0}^{T} \rho_{1}(s) d s, \\
y(T)+y^{\prime}(T)=\int_{0}^{T} \rho_{2}(s) d s .
\end{gathered}
$$

Lemma 3.2 (see [11]). Let $1<r \leq 2$ and let $\sigma, \rho_{1}, \rho_{2}: J \rightarrow E$ be continuous. A function $y$ is a solution of the fractional integral equation

$$
y(t)=P(t)+\int_{0}^{T} G(t, s) \sigma(s) d s
$$

with

$$
\begin{aligned}
P(t) & =\frac{(T+1-t)}{T+2} \int_{0}^{T} \rho_{1}(s) d s+\frac{(t+1)}{T+2} \int_{0}^{T} \rho_{2}(s) d s, \\
G(t, s) & = \begin{cases}\frac{(t-s)^{r-1}}{\Gamma(r)}-\frac{(1+t)(T-s)^{r-1}}{(T+2) \Gamma(r)}-\frac{(1+t)(T-s)^{r-2}}{(T+2) \Gamma(r-1)}, & 0 \leq s \leq t, \\
-\frac{(1+t)(T-s)^{r-1}}{(T+2) \Gamma(r)}-\frac{(1+t)(T-s)^{r-2}}{(T+2) \Gamma(r-1)}, & t \leq s \leq T,\end{cases}
\end{aligned}
$$

if and only if $y$ is a solution of the fractional boundary value problem (3.1).

Remark 3.3. It is clear that the function $t \mapsto \int_{0}^{T}|G(t, s)| d s$ is continuous on $J$, and hence is bounded. Let

$$
\tilde{G}:=\sup \left\{\int_{0}^{T}|G(t, s)| d s, t \in J\right\} .
$$


For the forthcoming analysis, we introduce the following assumptions

(H1) The functions $f, g, h: J \times E \rightarrow E$ satisfy the Carathéodory conditions.

(H2) There exist $p_{f}, p_{g}, p_{h} \in L^{\infty}\left(J, \mathbb{R}_{+}\right)$, such that

$$
\begin{aligned}
& \|f(t, y)\| \leq p_{f}(t)\|y\| \quad \text { for a.e. } t \in J \text { and each } y \in E, \\
& \|g(t, y)\| \leq p_{g}(t)\|y\|, \quad \text { for a.e. } t \in J \text { and each } y \in E, \\
& \|h(t, y)\| \leq p_{h}(t)\|y\|, \quad \text { for a.e. } t \in J \text { and each } y \in E .
\end{aligned}
$$

(H3) For almost each $t \in J$ and each bounded set $B \subset E$ we have

$$
\begin{aligned}
& \lim _{k \rightarrow 0^{+}} \alpha\left(f\left(J_{t, k} \times B\right)\right) \leq p_{f}(t) \alpha(B), \\
& \lim _{k \rightarrow 0^{+}} \alpha\left(g\left(J_{t, k} \times B\right)\right) \leq p_{g}(t) \alpha(B), \\
& \lim _{k \rightarrow 0^{+}} \alpha\left(h\left(J_{t, k} \times B\right)\right) \leq p_{h}(t) \alpha(B) .
\end{aligned}
$$

Theorem 3.4. Assume that assumptions (H1)-(H3) hold. If

$$
\frac{T(T+1)}{T+2}\left[\left\|p_{g}\right\|_{L^{\infty}}+\left\|p_{h}\right\|_{L^{\infty}}\right]+\tilde{G}\left\|p_{f}\right\|_{L^{\infty}}<1,
$$

then the boundary value problem (1.1) has at least one solution.

Proof. We transform the problem (1.1) into a fixed point problem by defining an operator $N: C(J, E) \rightarrow C(J, E)$ as

$$
(N y)(t)=P_{y}(t)+\int_{0}^{T} G(t, s) f(s, y(s)) d s,
$$

where

$$
P_{y}(t)=\frac{(T+1-t)}{T+2} \int_{0}^{T} g(s, y(s)) d s+\frac{(t+1)}{T+2} \int_{0}^{T} h(s, y(s)) d s,
$$

and the function $G(t, s)$ is given by (3.4). Clearly, the fixed points of the operator $N$ are solution of the problem (1.1). Let $R>0$ and consider the set

$$
D_{R}=\left\{y \in C(J, E):\|y\|_{\infty} \leq R\right\}
$$

Clearly, the subset $D_{R}$ is closed, bounded, and convex. We will show that $N$ satisfies the assumptions of Theorem 2.5. The proof will be given in three steps. 
Step 1. $N$ is continuous.

Let $\left\{y_{n}\right\}$ be a sequence such that $y_{n} \rightarrow y$ in $C(J, E)$. Then, for each $t \in J$,

$$
\begin{aligned}
\left|\left(N y_{n}\right)(t)-(N y)(t)\right| \leq & \frac{T+1}{T+2} \int_{0}^{T}\left\|g\left(s, y_{n}(s)\right)-g(s, y(s))\right\| d s \\
& +\frac{T+1}{T+2} \int_{0}^{T}\left\|h\left(s, y_{n}(s)\right)-h(s, y(s))\right\| d s \\
& +\int_{0}^{T}|G(t, s)|\left\|f\left(s, y_{n}(s)\right)-f(s, y(s))\right\| d s .
\end{aligned}
$$

Let $\rho>0$ be such that

$$
\left\|y_{n}\right\|_{\infty} \leq \rho, \quad\|y\|_{\infty} \leq \rho
$$

By $(\mathrm{H} 2)$ we have

$$
\begin{gathered}
\left\|g\left(s, y_{n}(s)\right)-g(s, y(s))\right\| \leq 2 \rho p_{g}(s):=\sigma_{1}(s) ; \quad \sigma_{1} \in L^{1}\left(J, \mathbb{R}_{+}\right), \\
\left\|h\left(s, y_{n}(s)\right)-h(s, y(s))\right\| \leq 2 \rho p_{h}(s):=\sigma_{2}(s) ; \quad \sigma_{2} \in L^{1}\left(J, \mathbb{R}_{+}\right), \\
|G(\cdot, s)|\left\|f\left(s, y_{n}(s)\right)-f(s, y(s))\right\| \leq 2 \rho|G(\cdot, s)| p_{f}(s):=\sigma_{3}(s) ; \quad \sigma_{3} \in L^{1}\left(J, \mathbb{R}_{+}\right) .
\end{gathered}
$$

Since $f, g$, and $h$ are Carathéodory functions, the Lebesgue dominated convergence theorem implies that

$$
\left\|N\left(y_{n}\right)-N(y)\right\|_{\infty} \longrightarrow 0 \text { as } n \longrightarrow \infty
$$

Step 2. $N$ maps $D_{R}$ into itself.

For each $y \in D_{R}$, by (H2) and (3.8) we have for each $t \in J$

$$
\begin{aligned}
\|N(y)(t)\| \leq & \frac{T+1}{T+2} \int_{0}^{T}\|g(s, y(s))\| d s+\frac{T+1}{T+2} \int_{0}^{T}\|h(s, y(s))\| d s \\
& +\int_{0}^{T}|G(t, s)|\|f(s, y(s))\| d s \\
\leq & R\left[\frac{T(T+1)}{T+2}\left\|p_{g}\right\|_{L^{\infty}}+\frac{T(T+1)}{T+2}\left\|p_{h}\right\|_{L^{\infty}}+\tilde{G}\left\|p_{f}\right\|_{L^{\infty}}\right] \\
< & R .
\end{aligned}
$$

Step 3. $N\left(D_{R}\right)$ is bounded and equicontinuous.

By Step 2, it is obvious that $N\left(D_{R}\right) \subset C(J, E)$ is bounded. 
For the equicontinuity of $N\left(D_{R}\right)$. Let $t_{1}, t_{2} \in J, t_{1}<t_{2}$ and $y \in D_{R}$. Then

$$
\begin{aligned}
\left\|(N y)\left(t_{2}\right)-(N y)\left(t_{1}\right)\right\|= & \|-\frac{t_{2}-t_{1}}{T+2} \int_{0}^{T} g(s, y(s)) d s+\frac{t_{2}-t_{1}}{T+2} \int_{0}^{T} h(s, y(s)) d s \\
& +\int_{0}^{T}\left[G\left(t_{2}, s\right)-G\left(t_{1}, s\right)\right] f(s, y(s)) d s \| \\
\leq & \frac{t_{2}-t_{1}}{T+2} T R\left[\left\|p_{g}\right\|_{L^{\infty}}+\left\|p_{h}\right\|_{L^{\infty}}\right] \\
& +R\left\|p_{f}\right\|_{L^{\infty}} \int_{0}^{T}\left|G\left(t_{2}, s\right)-G\left(t_{1}, s\right)\right| d s .
\end{aligned}
$$

As $t_{1} \rightarrow t_{2}$, the right-hand side of the above inequality tends to zero.

Now let $V$ be a subset of $D_{R}$ such that $V \subset \overline{c O}(N(V) \cup\{0\})$.

$V$ is bounded and equicontinuous, and therefore the function $v \rightarrow v(t)=\alpha(V(t))$ is continuous on $J$. By (H3), Lemma 2.6, and the properties of the measure $\alpha$ we have for each $t \in J$

$$
\begin{aligned}
v(t) \leq & \alpha(N(V)(t) \cup\{0\}) \\
\leq & \alpha(N(V)(t)) \\
\leq & \int_{0}^{T}\left|\frac{T+1-t}{T+2}\right| p_{g}(s) \alpha(V(s)) d s+\int_{0}^{T}\left|\frac{t+1}{T+2}\right| p_{h}(s) \alpha(V(s)) d s \\
& +\int_{0}^{T}|G(t, s)| p_{f}(s) \alpha(V(s)) d s \\
\leq & \frac{T(T+1)}{T+2}\left\|p_{g}\right\|_{L^{\infty}} v(s)+\frac{T(T+1)}{T+2}\left\|p_{h}\right\|_{L^{\infty}} v(s)+\tilde{G}\left\|p_{f}\right\|_{L^{\infty}} v(s) \\
\leq & \|v\|_{\infty}\left[\frac{T(T+1)}{T+2}\left[\left\|p_{g}\right\|_{L^{\infty}}+\left\|p_{h}\right\|_{L^{\infty}}\right]+\tilde{G}\left\|p_{f}\right\|_{L^{\infty}}\right] .
\end{aligned}
$$

This means that

$$
\|v\|_{\infty}\left(1-\left[\frac{T(T+1)}{T+2}\left[\left\|p_{g}\right\|_{L^{\infty}}+\left\|p_{h}\right\|_{L^{\infty}}\right]+\tilde{G}\left\|p_{f}\right\|_{L^{\infty}}\right]\right) \leq 0
$$

By (3.8) it follows that $\|v\|_{\infty}=0$, that is, $v(t)=0$ for each $t \in J$, and then $V(t)$ is relatively compact in $E$. In view of the Ascoli-Arzelà theorem, $V$ is relatively compact in $D_{R}$. Applying now Theorem 2.5 we conclude that $N$ has a fixed point which is a solution of the problem (1.1). 


\section{An Example}

In this section we give an example to illustrate the usefulness of our main results. Let us consider the following fractional boundary value problem:

$$
\begin{aligned}
{ }^{c} D^{r} y(t) & =\frac{2}{19+e^{t}}|y(t)|, \quad t \in J:=[0,1], 1<r \leq 2, \\
y(0)-y^{\prime}(0) & =\int_{0}^{1} \frac{1}{5+e^{5 s}}|y(s)| d s, \\
y(1)+y^{\prime}(1) & =\int_{0}^{1} \frac{1}{3+e^{3 s}}|y(s)| d s .
\end{aligned}
$$

Set

$$
\begin{aligned}
& f(t, x)=\frac{2}{19+e^{t}} x, \quad(t, x) \in J \times[0, \infty), \\
& g(t, x)=\frac{1}{5+e^{5 t}} x, \quad(t, x) \in[0,1] \times[0, \infty), \\
& h(t, x)=\frac{1}{3+e^{3 t}} x, \quad(t, x) \in[0,1] \times[0, \infty) .
\end{aligned}
$$

Clearly, conditions (H1),(H2) hold with

$$
p_{f}(t)=\frac{2}{19+e^{t}}, \quad p_{g}(t)=\frac{1}{5+e^{5 t}}, \quad p_{h}(t)=\frac{1}{3+e^{3 t}} .
$$

From (3.4) the function $G$ is given by

$$
G(t, s)= \begin{cases}\frac{(t-s)^{r-1}}{\Gamma(r)}-\frac{(1+t)(1-s)^{r-1}}{3 \Gamma(r)}-\frac{(1+t)(1-s)^{r-2}}{3 \Gamma(r-1)}, & 0 \leq s \leq t, \\ -\frac{(1+t)(1-s)^{r-1}}{3 \Gamma(r)}-\frac{(1+t)(1-s)^{r-2}}{3 \Gamma(r-1)}, & t \leq s \leq 1 .\end{cases}
$$

From (4.4), we have

$$
\begin{aligned}
\int_{0}^{1} G(t, s) d s= & \int_{0}^{t} G(t, s) d s+\int_{t}^{1} G(t, s) d s \\
= & \frac{t^{r}}{\Gamma(r+1)}+\frac{(1+t)(1-t)^{r}}{3 \Gamma(r+1)}-\frac{(1+t)}{3 \Gamma(r+1)} \\
& +\frac{(1+t)(1-t)^{r-1}}{3 \Gamma(r)}-\frac{(1+t)}{3 \Gamma(r)} \\
& -\frac{(1+t)(1-t)^{r}}{3 \Gamma(r+1)}-\frac{(1+t)(1-t)^{r-1}}{3 \Gamma(r)} .
\end{aligned}
$$


A simple computation gives

$$
G^{*}<\frac{3}{\Gamma(r+1)}+\frac{2}{\Gamma(r)}
$$

Condition (3.8) is satisfied with $T=1$. Indeed

$$
\begin{aligned}
\frac{T(T+1)}{T+2}\left[\left\|p_{g}\right\|_{L^{\infty}}+\left\|p_{h}\right\|_{L^{\infty}}\right]+\tilde{G}\left\|p_{f}\right\|_{L^{\infty}} & <\frac{2}{3}\left[\frac{1}{6}+\frac{1}{4}\right]+\frac{3}{10 \Gamma(r+1)}+\frac{2}{10 \Gamma(r)} \\
& =\frac{5}{18}+\frac{3}{10 \Gamma(r+1)}+\frac{1}{5 \Gamma(r)}<1
\end{aligned}
$$

which is satisfied for each $r \in(1,2]$. Then by Theorem 3.4 the problem (4.1) has a solution on $[0,1]$.

\section{Acknowledgments}

The authors thank the referees for their remarks. The research of A. Cabada has been partially supported by Ministerio de Educacion y Ciencia and FEDER, project MTM2007-61724, and by Xunta de Galicia and FEDER, project PGIDIT05PXIC20702PN.

\section{References}

[1] R. Hilfer, Ed., Applications of Fractional Calculus in Physics, World Scientific, River Edge, NJ, USA, 2000.

[2] W. G. Glockle and T. F. Nonnenmacher, "A fractional calculus approach to self-similar protein dynamics," Biophysical Journal, vol. 68, no. 1, pp. 46-53, 1995.

[3] R. Metzler, W. Schick, H.-G. Kilian, and T. F. Nonnenmacher, "Relaxation in filled polymers: a fractional calculus approach," The Journal of Chemical Physics, vol. 103, no. 16, pp. 7180-7186, 1995.

[4] I. Podlubny, Fractional Differential Equations, vol. 198 of Mathematics in Science and Engineering, Academic Press, San Diego, Calif, USA, 1999.

[5] L. Gaul, P. Klein, and S. Kemple, "Damping description involving fractional operators," Mechanical Systems and Signal Processing, vol. 5, no. 2, pp. 81-88, 1991.

[6] R. P. Agarwal, M. Benchohra, and S. Hamani, "Boundary value problems for differential inclusions with fractional order," Advanced Studies in Contemporary Mathematics, vol. 16, no. 2, pp. 181-196, 2008.

[7] B. Ahmad and J. J. Nieto, "Existence results for nonlinear boundary value problems of fractional integrodifferential equations with integral boundary conditions," Boundary Value Problems, vol. 2009, Article ID 708576, 11 pages, 2009.

[8] B. Ahmad and V. Otero-Espinar, "Existence of solutions for fractional differential inclusions with antiperiodic boundary conditions," Boundary Value Problems, vol. 2009, Article ID 625347, 11 pages, 2009.

[9] A. Belarbi, M. Benchohra, and A. Ouahab, "Uniqueness results for fractional functional differential equations with infinite delay in Fréchet spaces," Applicable Analysis, vol. 85, no. 12, pp. 1459-1470, 2006.

[10] M. Belmekki, J. J. Nieto, and R. R. Rodriguez-Lopez, "Existence of periodic solution for a nonlinear fractional differential equation," Boundary Value Problems. in press.

[11] M. Benchohra, J. R. Graef, and S. Hamani, "Existence results for boundary value problems with nonlinear fractional differential equations," Applicable Analysis, vol. 87, no. 7, pp. 851-863, 2008.

[12] M. Benchohra, S. Hamani, and S. K. Ntouyas, "Boundary value problems for differential equations with fractional order," Surveys in Mathematics and Its Applications, vol. 3, pp. 1-12, 2008.

[13] M. Benchohra, J. Henderson, S. K. Ntouyas, and A. Ouahab, "Existence results for fractional order functional differential equations with infinite delay," Journal of Mathematical Analysis and Applications, vol. 338, no. 2, pp. 1340-1350, 2008. 
[14] Y.-K. Chang and J. J. Nieto, "Some new existence results for fractional differential inclusions with boundary conditions," Mathematical and Computer Modelling, vol. 49, no. 3-4, pp. 605-609, 2009.

[15] V. Daftardar-Gejji and S. Bhalekar, "Boundary value problems for multi-term fractional differential equations," Journal of Mathematical Analysis and Applications, vol. 345, no. 2, pp. 754-765, 2008.

[16] R. Figueiredo Camargo, A. O. Chiacchio, and E. Capelas de Oliveira, "Differentiation to fractional orders and the fractional telegraph equation," Journal of Mathematical Physics, vol. 49, no. 3, Article ID 033505, 12 pages, 2008.

[17] A. A. Kilbas, H. M. Srivastava, and J. J. Trujillo, Theory and Applications of Fractional Differential Equations, vol. 204 of North-Holland Mathematics Studies, Elsevier Science B.V., Amsterdam, The Netherlands, 2006.

[18] N. Heymans and I. Podlubny, "Physical interpretation of initial conditions for fractional differential equations with Riemann-Liouville fractional derivatives," Rheologica Acta, vol. 45, no. 5, pp. 765-771, 2006.

[19] I. Podlubny, "Geometric and physical interpretation of fractional integration and fractional differentiation," Fractional Calculus E Applied Analysis for Theory and Applications, vol. 5, no. 4, pp. 367-386, 2002.

[20] K. W. Blayneh, "Analysis of age-structured host-parasitoid model," Far East Journal of Dynamical Systems, vol. 4, no. 2, pp. 125-145, 2002.

[21] G. Adomian and G. E. Adomian, "Cellular systems and aging models," Computers $\mathcal{E}$ Mathematics with Applications, vol. 11, no. 1-3, pp. 283-291, 1985.

[22] A. Arara and M. Benchohra, "Fuzzy solutions for boundary value problems with integral boundary conditions," Acta Mathematica Universitatis Comenianae, vol. 75, no. 1, pp. 119-126, 2006.

[23] M. Benchohra, S. Hamani, and J. Henderson, "Functional differential inclusions with integral boundary conditions," Electronic Journal of Qualitative Theory of Differential Equations, vol. 2007, no. 15, pp. 1-13, 2007.

[24] M. Benchohra, S. Hamani, and J. J. Nieto, "The method of upper and lower solutions for second order differential inclusions with integral boundary conditions," to appear in The Rocky Mountain Journal of Mathematics.

[25] G. Infante, "Eigenvalues and positive solutions of ODEs involving integral boundary conditions," Discrete and Continuous Dynamical Systems, pp. 436-442, 2005.

[26] S. Peciulyte, O. Stikoniene, and A. Stikonas, "Sturm-Liouville problem for stationary differential operator with nonlocal integral boundary condition," Mathematical Modelling and Analysis, vol. 10, no. 4, pp. 377-392, 2005.

[27] J. Banaś and K. Goebel, Measures of Noncompactness in Banach Spaces, vol. 60 of Lecture Notes in Pure and Applied Mathematics, Marcel Dekker, New York, NY, USA, 1980.

[28] J. Banas and K. Sadarangani, "On some measures of noncompactness in the space of continuous functions," Nonlinear Analysis: Theory, Methods E Applications, vol. 68, no. 2, pp. 377-383, 2008.

[29] D. Guo, V. Lakshmikantham, and X. Liu, Nonlinear Integral Equations in Abstract Spaces, vol. 373 of Mathematics and Its Applications, Kluwer Academic Publishers, Dordrecht, The Netherlands, 1996.

[30] V. Lakshmikantham and S. Leela, Nonlinear Differential Equations in Abstract Spaces, vol. 2 of International Series in Nonlinear Mathematics: Theory, Methods and Applications, Pergamon Press, Oxford, UK, 1981.

[31] H. Mönch, "Boundary value problems for nonlinear ordinary differential equations of second order in Banach spaces," Nonlinear Analysis: Theory, Methods \& Applications, vol. 4, no. 5, pp. 985-999, 1980.

[32] S. Szufla, "On the application of measure of noncompactness to existence theorems," Rendiconti del Seminario Matematico della Università di Padova, vol. 75, pp. 1-14, 1986.

[33] R. P. Agarwal, M. Meehan, and D. O'Regan, Fixed Point Theory and Applications, vol. 141 of Cambridge Tracts in Mathematics, Cambridge University Press, Cambridge, UK, 2001. 